News \& Views in ...

\title{
Personalized Medicine
}

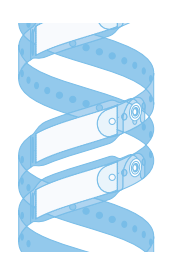

Highlighting the latest news in personalized medicine

\section{Predicting the future with a mathematical model}

Researchers from the Dana-Faber Cancer Institute (MA, USA) have developed a mathematical model capable of predicting future behavior of a patient's tumor and which treatments are most likely to be effective.

In the study, published in the journal Cell Reports, researchers combined several data types from pre- and post-treatment biopsies of breast tumors to obtain a molecular picture of how the cancer evolved as a result of chemotherapy. The study included samples from 47 patients who underwent preoperative chemotherapy.

The resulting predictive model was generated by integrating data on genetic and other traits of numerous individual cells within the tumor sample along with maps detailing where the cells were located within the tumors.

"Better understanding of tumor evolution is key to improving the design of cancer therapies and for truly individualized cancer treatment," explained Kornelia Polyak of the Dana-Farber Institute. "We asked two questions - how heterogeneity influences treatment outcomes and how treatment changes heterogeneity," she continued.

The computer model produced some general findings. The genetic diversity within a tumor did not alter much in can-

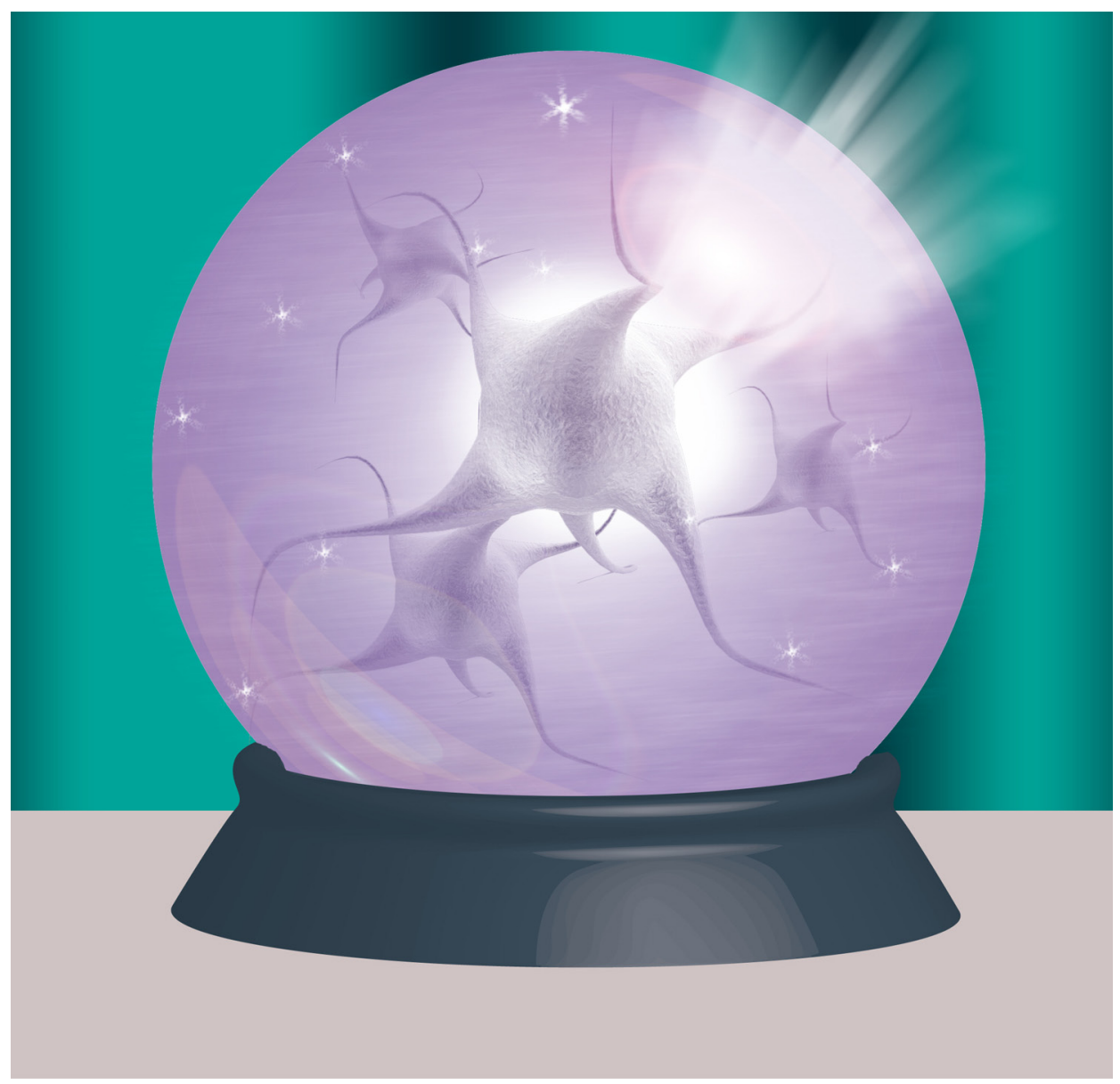


cers exhibiting no response or only partial response to treatment. In addition, tumors with less genetic diversity were calculated to be more likely to completely respond to treatment than tumors with more genetic complexity.
The authors of the study hope that in the future their model may be used by clinicians to analyze a patient's tumor at the point of diagnosis. The results of the analysis could help tailor specific drugs and plan treatment strategies to match the tumor's predicted behavior.

- Written by Hannah Wilson

Sources: Almendro V, Cheng YK, Randles A et al. Inference of tumor evolution during chemotherapy by computational modeling and in situ analysis of genetic and phenotypic cellular diversity. Cell Rep. doi:10.1016/j.celrep.2013.12.041 (2014) (Epub ahead of print); Dana-Farber Cancer Institute press release: www.dana-farber.org/Newsroom/News-Releases/new-computer-mode/may-aid-personalized-cancer-care.aspx

\section{Call for donors to have access to their biobank data}

A recent Policy Forum paper published in the journal Science has called for data held in biobanks to be made accessible to donors. The paper, written by researchers from Harvard Medical School (MA, USA) and King's College London (UK), suggests that donors should be allowed unrestricted access to data derived from their own material, an option entirely possible with current technology. At present, data held in biobanks are only accessible to researchers and not to the individuals who contributed the materials.

Explaining the basis for the paper, coauthor and member of the Personalized Medicine editorial board Barbara Prainsack (King's College London) stated: "My colleagues and I are following the interesting discussions that are going on at the moment about when and how to return incidental findings in both clinical and research contexts. While we feel that these discussions are very important, they are focusing on only one end of the pipeline, namely at the stage when analyses and interpretations have already been done. In our mind we also need to talk about the other end of the pipeline, namely at the point of data entry: when data - those derived from samples, clinical data, imaging data, et cetera - are entered into a system, then people should have access to their own data, if they so desire. In other words, our point is about giving people access to their own 'raw' data before analyses have been carried out. It is important to us to emphasize that our argument is not that people should make use of this possibility; we are saying something about the importance of giving them the opportunity. We expect that many people will not want their raw data, because they have no use for them."

The authors explain the importance of the idea as a key step in treating donors as true research participants. In addition, the need for access to be arranged from the point of study design/initiation, with important consequences for both protocol and consent is discussed.

Speaking to Personalized Medicine, Prainsack gave her vision for the future of this contentious issue, stating: "The next step will be to spell out the practicalities and implications of implementing this concept. We have been thinking about this - together with colleagues in various disciplines - and we will continue to collaborate on this."

\section{Telecounseling: an effective alternative?}

Genetic counseling delivered via telephone is as effective as face-to-face counseling according to a study published in Journal of Clinical Oncology.

The multicenter study, led by investigators from Georgetown Lombardi Comprehensive Cancer Center (Georgetown University, DC, USA), is the largest randomized comparison of the two methods to date.

Explaining the importance of genetic counseling in general, and this study in particular, lead investigator
Marc Schwartz (Georgetown University) stated: "It's important that all people interested in testing have access to thorough information so they can consider the implications of test results and interpret them in the context of family history. Counseling on the phone reduces costs and expands genetic testing access to rural areas, where counseling isn't always available."

The study was conducted with women considering testing for mutations in BRCA1 and/or BRCA2, but 
the investigators hope their findings may be extended to other hereditary cancers and other conditions. Researchers randomized 669 women to receive either telephone or face-to-face counseling. Notably, approximately a third of women originally approached about participating in the study declined owing to not wanting to receive counseling via telephone. The majority of study participants had been treated for breast or ovarian cancer and the rest were highlighted as being at risk for the disease due to identification of a $B R C A$ mutation in a family member.

Patients received counseling before and after genetic testing, and were surveyed on numerous variables, including their understanding of the test, perceived stress, satisfaction and decision conflict. The survey results found that telephone counseling was just as effective as the face-to-face alternative.

Speaking about the group's work, coauthor Beth Peshkin (Georgetown University) explained: "We hypothesized that telephone counseling would be comparable to in-person counseling overall and would be preferable for some participants. It's important for us to learn more about why people prefer one type of counseling over another, and how that affects use and outcomes of genetic counseling."

\section{Myriad test validated in approximately 5000 patients}

Myriad Genetics, Inc. has presented clinical data showing their myRisk ${ }^{\mathrm{TM}}$ Hereditary Cancer test to identify $51 \%$ more patients with a higher risk of hereditary breast and ovarian cancer than BRCA1/2 testing alone.

myRisk uses next-generation sequencing technology to evaluate 25 genes associated with eight major hereditary cancers: breast, colorectal, ovarian, endometrial, pancreatic, prostate and gastric cancer, and melanoma.

Data from a large prospective clinical validation study were presented at the 2013 San Antonio Breast Cancer Symposium (TX, USA). The study measured mutations in 25 cancer-causing genes in patients referred for BRCA1/2 testing. Of the 1951 patients evaluated, 257 tested positive for a deleterious mutation with the myRisk test. Testing for BRCA1/2 genes alone identified only 182 of the mutation carriers, represent- ing a $51 \%$ increase in breast and ovarian cancer mutation detection when using the myRisk test. Accuracy of the test was calculated at $99.99 \%$.

The myRisk Hereditary cancer test has now been validated in approximately 5000 patients across numerous clinical studies.

"Hereditary cancers are caused by mutations in many different genes and new genes linked to cancer have been recently identified. Testing only for one hereditary cancer syndrome may lead to missed mutations," explained Myriad's chief medical officer Richard J Wenstrup. "The myRisk test evaluates 25 clinically actionable genes and significantly improves our ability to identify people at higher risk for hereditary cancer, which may lead to prevention or early detection with the ability to reduce cancer incidence and mortality."

\section{Genomic study identifies higher responses and improved survival outcomes in HER2+ breast cancer}

Research led by the Translational Genomics Group at Valld'Hebron Institute of Oncology (VHIO; Barcelona, Spain) has not only shown that HER2 ${ }^{+}$breast cancer can be classified into four different subtypes, but has also unmasked a subtype showing a greater response to and increased benefit from chemotherapy and anti-HER2 therapy. It is hoped that this newly refined classification will facilitate more effective treatments tailored specific tumors, as well as advance targeted therapy against HER $2^{+}$breast cancer.

Not all HER $2^{+}$tumors respond in the same way to anti-HER2 targeted therapy; as such, researchers at VHIO conducted a retrospective study of patients treated in the Phase III NOAH clinical trial, with the 
aim of establishing the genomic differences of both the treatment-sensitive and the treatment-resistant tumors.

From 114 pretreated patients, gene-expression profiling was performed using RNA from formalin-fixed paraffin-embedded core biopsies, with HER2 $2^{+}$tumors randomized to receive neoadjuvant doxorubicin/paclitaxel followed by cyclophosphamide/methotrexate/ fluorouracil, or the same regimen in combination with trastuzumab for 1 year. A control cohort of 42 patients was also included.

The prediction analysis of microarray 50 (PAM50) subtypes, the PAM50 proliferation score, and the PAM50 risk of relapse score based on subtype and subtype and proliferation (RORP) were evaluated. Results showed that HER2-enriched (HER2-E) tumors predominated within HER2 ${ }^{+}$disease, although all PAM50 intrinsic subtypes were identified across the three cohorts. The odds ratio for achieving pathologic complete response with trastuzumab-based chemotherapy for HER2 $2^{+}$HER2-E and HER2 ${ }^{+} /$RORP-high was $5.117(\mathrm{p}=0.009)$ and $8.469(\mathrm{p}=0.025)$, respectively, compared with chemotherapy only.

"We have found that HER 2+ tumors in the HER2-E subtype have a highly activated HER2 signaling pathway, thereby making them especially sensitive to anti-HER2 targeted therapies such as trastuzumab," explained Aleix Prat, principal investigator of VHIO's Translational Genomics Group. "Patients with HER2 + HER2-E tumors, or HER2+/RORP-high tumors, benefit substantially from trastuzumab-based chemotherapy," the authors concluded.

- Written by Ruth Williamson

Sources: Prat A, Bianchini G, Thomas M et al. Research-based PAM50 subtype predictor identifies higher responses and improved survival outcomes in HER2-positive breast cancer in the NOAH Study. Clin. Cancer Res. 20(2), 511-521 (2014); VHIO press release: www.vhio.net/news/138/vhio-genomic-study-identifies-subgroups-of-her2-breast-cancer-with-varying-sensitivities-toanti-her2-treatment 